\title{
New Electron Holographic Technique for the Measurement of Strain at the Nanoscale: Application to Electronic Devices and Multilayers
}

Etienne Snoeck, Florent Houdellier, Florian Hüe, and Martin Hÿtch

CEMES-CNRS, 29 rue Jeanne Marvig, 31055 Toulouse, France

We present details of the new electron holographic dark-field technique for mapping strain in nanostructures [1]. Experiments are carried out in a transmission electron microscope (TEM) equipped with a highly coherent electron source (FEG). The method combines the advantages of the conventional moire technique with the flexibility of off-axis electron holography and is applicable to standard focused-ion beam (FIB) prepared samples. It consists in making the interference with the aid of an electrostatic biprism between a diffracted beam emanating from an unstrained region (region " $A$ " in Fig. 1) of crystal with a similar "g" diffracted beam coming from a strained region of interest ("B").

Geometric phase analysis (GPA) [2] of the holographic fringes allows determining the relative deformation of the two crystalline lattices. Strain can therefore be measured to high precision, with nanometre spatial resolution and for micron fields of view. The method can be applied on any deformed crystalline materials (epitaxial multilayers, precipitates in alloys, strained devices) provided that the material is crystalline and that a reference area is located in the vicinity of the strained region of interest.

We will present measurements of strain in the active regions of strained-silicon MOSFET devices (Fig.2a) and compare the results with finite element modelling (Fig. 2b) [1,3]. We also will present results on deformation measurements on InAs/GaInAs epitaxial multilayers.

The current limitations of the technique will be discussed along with future areas of application.

\section{References}

[1] M.J. Hÿtch, F. Houdellier, F. Hüe, and E. Snoeck, Nature 453 (2008) 1086-1089.

doi:10.1038/nature07049.

[2] M.J. Hÿtch, E. Snoeck and R. Kilaas, Ultramicroscopy 74 (1998) 131-146.

[3] F. Hüe, M.J. Hÿtch, F. Houdellier, H. Bender, and A. Claverie, Appl. Phys. Lett. 95, doi: $10.1063 / 1.3192356$.

This work was supported by the European Commission through the ESTEEM project (Enabling Science and Technology for European Electron Microscopy, Grant No. IP3:0260019) and by the French National Agency (ANR) in the frame of its programin Nanosciences and Nanotechnologies (HD STRAIN Project No. ANR-08-NANO-0 32). 


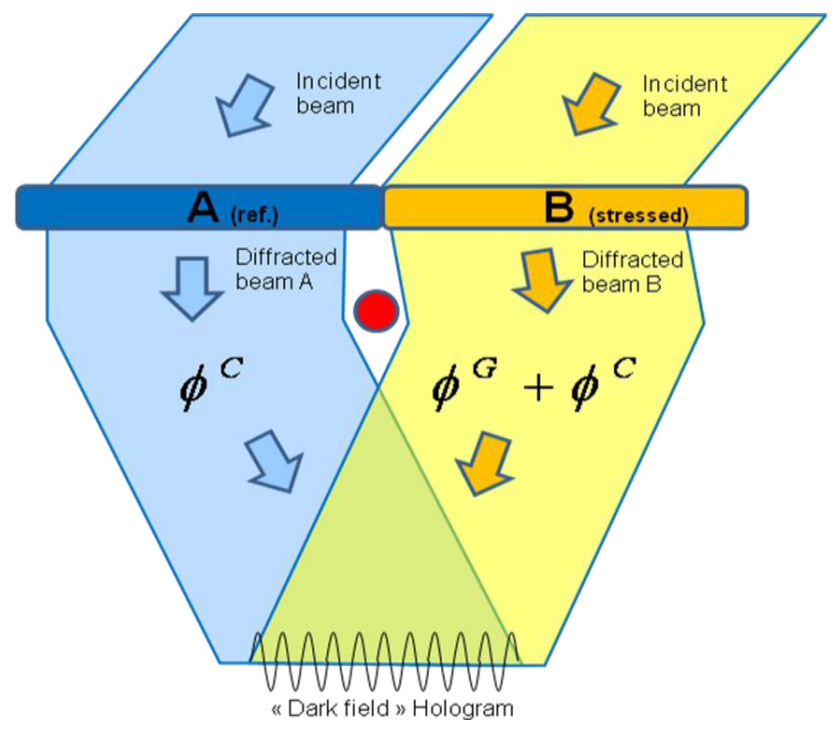

Fig. 1: Scheme of the "Dark-holo" configuration

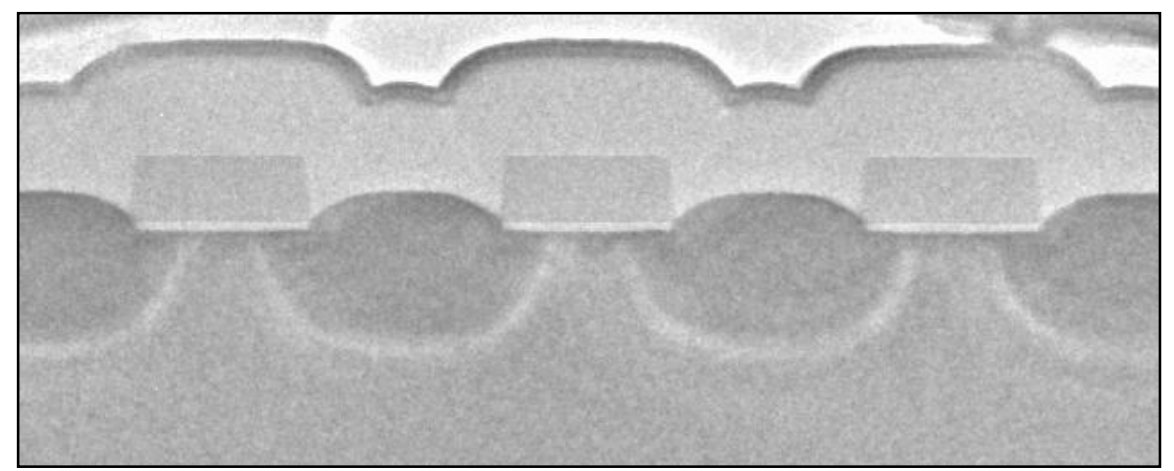

Fig. 2a: TEM micrograph of three p-MOS transistors constituted of strained-Si channels compressed by embedded SiGe sources and drain
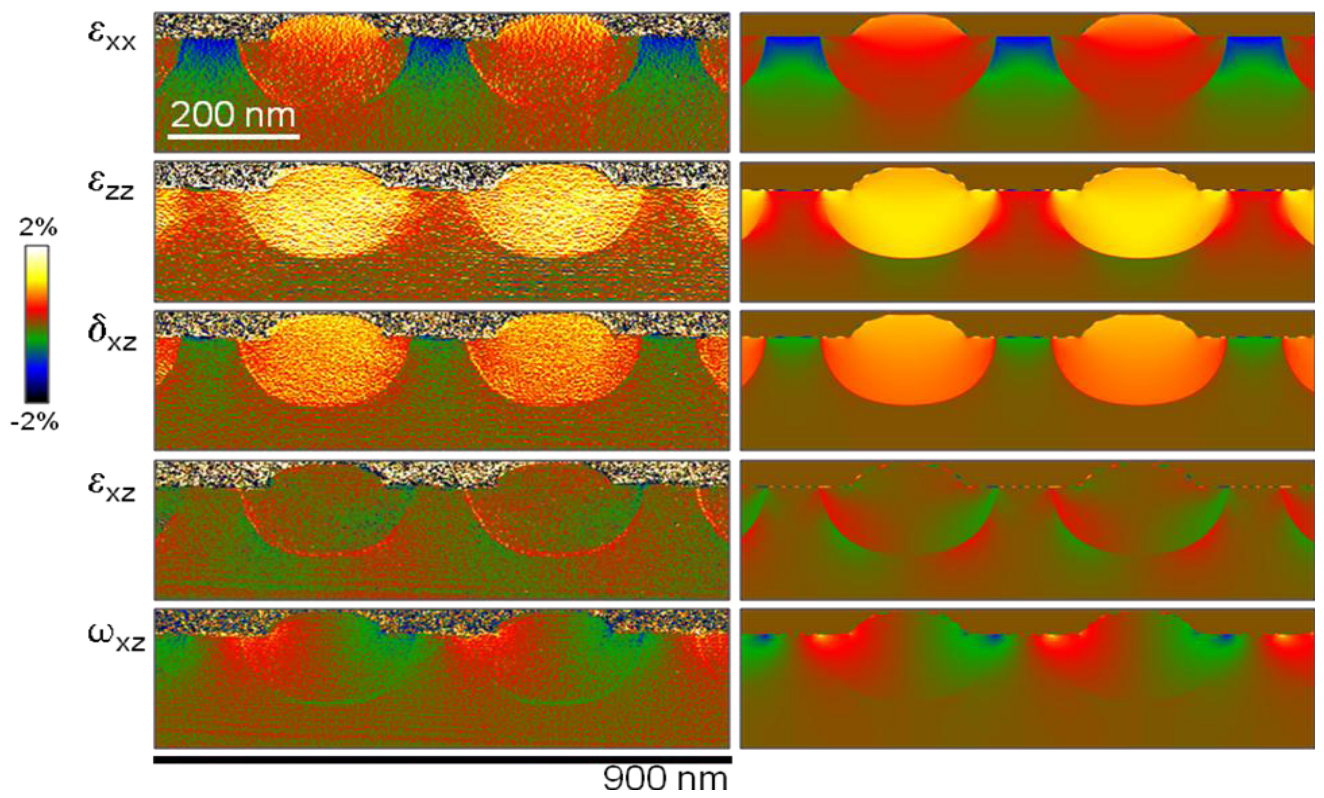

Fig 2b: Experimental (left) and simulated (right) maps of the deformations, shear and rotation 